\title{
DO REDUNDANT HEAD-UP AND HEAD-DOWN DISPLAY CONFIGURATIONS CAUSE DISTRACTIONS?
}

\author{
Carl Jörgen Normark, Phillip Tretten \& Anita Gärling \\ Department of Human Work Sciences \\ Luleå University of Technology \\ Luleå, Sweden \\ E-mail: jorgen.normark@1tu.se
}

\begin{abstract}
Summary: This study was designed to investigate effects of different display configuration designs. Nineteen drivers completed a driving simulator study designed to resemble normal driving. Driving performance, glance behaviour, physiological measures, and task completion times was measured for two display configuration designs both during driving only and during driving with a simple secondary task, which consisted of detection, and off-setting of presented warnings. The display configuration design with more centrally placed information, e.g. the HUD and HDD, had less detrimental effects on driving performance and glance behaviour. The physiological measures showed, however, no significant differences between display configuration designs.
\end{abstract}

\section{INTRODUCTION}

Automobiles are becoming more and more technologically complex (Baber \& Wankling, 1992; Noy, 1997) with more and more built-in driver information systems (Tsimhoni \& Green, 2001). This also increases the amount, and range, of information presented to the driver, which leads to the use of dynamic displays showing many types of information in one place (Baber \& Wankling, 1992). The driver is mainly guided by vision for driving, and visual information can be lost in the quantity of information displayed to the driver and this can have a negative effect on driving safely since the visual faculties competes with the same perceptual and cognitive resources as the task of driving (Horberry, Anderson, Regan, Triggs, \& Brown, 2006). For a safe traffic environment, drivers must have their attention on the outside of the car rather than on invehicle displays (Baber \& Wankling, 1992). Luoma \& Rämä (2002) even claim that an invehicle information system should only provide information when it's really needed. Having attention on something other than driving, a secondary task, increases the in-vehicle glance durations and is associated with decreased driving performance and increased crash risk (Horrey \& Wickens, 2007; Tsimhoni \& Green, 2001). Horrey, Wickens, \& Consalus (2005) and Wittman et al. (2006) declare that if information is acquired from a display located close to the road view, the driving performance is less degraded than for a distant display. Using a Head-Up Display (HUD) can also result in reduced workload, decreased response times, and increased driving comfort (Liu \& Wen 2004). According to Wittmann et al., (2006), the detection of signals is easiest near the line of sight, and it decreases significantly for larger eccentricities, especially the vertical. According to Gish \& Staplin (1995), a HUD can increase the time the eyes are kept on the road, but also deteriorate the reaction time for events on the road by increased visual clutter in the driver's line of sight. According to Ellis (2005), redundant elements may aid in the interpretation of information, but other forms of redundancy may also deteriorate performance (Seagull, Wickens \& Loeb, 2001). 
Physiological measures have been used in earlier traffic safety studies and might be useful for studying workload in in-vehicle technology (Johansson et al., 2004).

Research aim and research questions. The aim of this study is to investigate the influence of two display configurations on driving performance and glance behaviour both while driving and while performing a simple secondary task while driving. One display configuration had a four display design similar to those found in high-end automobiles of today (e.g. BMW 7-series) while the other presented information centrally and redundant in two different displays; a LCD display in a HUD position $15^{\circ}$ from the drivers' normal line of sight, that did not obstruct the drivers view of the road, and a regular instrument cluster head-down display (HDD). The following specific questions are addressed: Does redundantly displayed information placed in the driver's line of sight differ with respect to driving performance, added distraction, and time spent looking away from the road? Is the driver's stress level affected by having information displayed in the line of sight? Are warnings detected and distinguished from normal in-vehicle information while driving?

\section{METHOD}

\section{Participants and equipment}

Nineteen drivers (10 males and 9 females aged 20 to 58 years with a mean age of 37.6 years) conducted the study. All participants had a valid driver's licence and either normal or corrected to normal vision. None of the participants' private vehicles were equipped with any type of HUD display. The experiment took place in Luleå University of Technology Designlab's driving simulator, consisting of a fixed base Volvo XC90 cockpit where four LCD displays replaced the original instrumentation (Figure 1) and it's handling was configured to simulate a front-wheel drive SUV (e.g., Volvo XC90). The road view was projected by a NEC NP-1000 projector on a $1.8 \mathrm{~m}$ high by $2.4 \mathrm{~m}$ wide screen in front of the driver which subtends about $33.4^{\circ}$ of the driver's forward view. Eye movements were monitored by Seeing Machine's FaceLab system (version 4.5). The minimum duration for a glance was in this study set to $100 \mathrm{~ms}$ (Horrey \& Wickens, 2007). Eye data calculations were based on fixations towards areas of interest (AOIs).

Physiological measures were collected with Mind Media's Nexus-10 hardware and BioTrace (version 1.20) software. EKG sensors in a Lead II chest position were used for measuring heart rate (HR). Galvanic skin resistance (GSR) sensors were mounted on the index and ring finger of the left hand. A temperature sensor was mounted on the middle finger on the left hand.

\section{Driving environment}

The driving environment was designed to simulate a realistic route with traffic, surroundings, and events that might occur in a realistic driving situation. Carsten et al. (2005) suggest that a rural road generally gives the largest effect sizes for a driving simulator study. In this study, an approximately $15 \mathrm{~km}$ long road with two lanes through rural areas and with a short four lane segment through a city environment was used. There were segments with 50 and $70 \mathrm{~km} / \mathrm{h}$ speed limits. Throughout the study there was some oncoming traffic in the opposite lane and some in the same lane as the driver to simulate realistic driving. To keep the driver focused on the driving, some cars had to be overtaken, some cars made unexpected manoeuvres by abruptly braking, and at one time a cyclist entered and crossed the road from behind a parked truck. 


\section{Experimental design}

The experiment was a 2 (driving condition) $\mathrm{x} 2$ (display configuration) factorial design with repeated measures on the first factor. The driving conditions were "driving only" and "driving with a task". For the latter warnings were presented to the driver in the form of common automobile warnings consisting of a $15 \times 15 \mathrm{~mm}$ icon accompanied by a text such as for instance "Low washer fluid". There were ten generic warnings of similar length and similar complicity displayed. The participants were instructed to offset the warnings as soon as they noticed them. The two display configurations were "Redundant HUD" where vehicle speed and warnings was presented to the driver redundant in the HUD and HDD, and "Spread out" where vehicle speed was presented in the HDD display and warnings appeared in one of the four display positions (Figure 1). The order of displays the warnings appeared in was: Infotainment display (IF), Centerstack (CS), HDD, IF, HUD, HDD, IF, HUD, HDD, and CS.
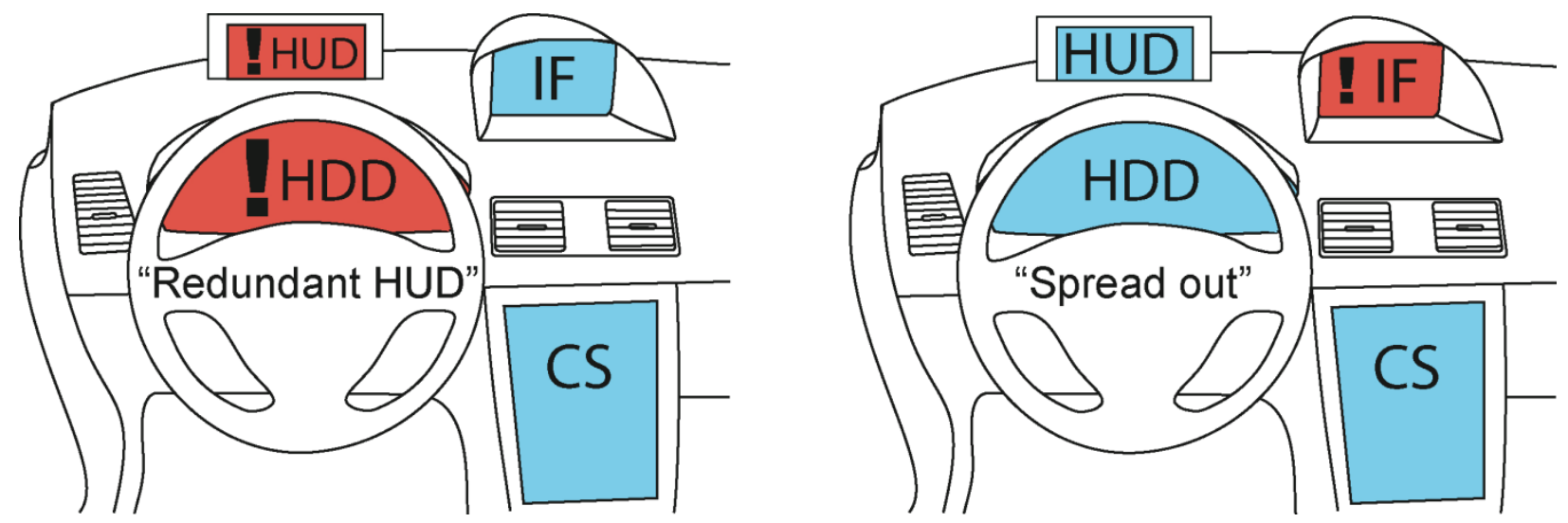

Figure 1. Display configurations. HUD - Head-up, HDD - Head-down, IF - Infotainment, CS - Centerstack

\section{Procedure}

The experimental session started with the participants being introduced to the simulator and given a five minute practice run to get familiar with handling the simulator. There were then two driving blocks of 15 minute each; a "driving only" block and a "driving and task" block. The order of these was balanced and both were made on the same road segment but in reverse directions in order to prevent the driver from getting too familiar with the road segment. The "driving only" block consisted only of driving through the road segment. The "driving and task" block consisted of driving with the addition of a simple secondary visual detection task, where the drivers were asked to, while driving, reset a total of ten warnings as soon as they had been discovered. The participants were asked to drive as they normally do with their own vehicles and to obey presented speed limits. Custom software was used to synchronize and reduce all data regarding the dependent measures (Table 2) to $10 \mathrm{~Hz}$, to analyze gaze data, driving data, and physiological data. Mann-Whitney's U-test with significance levels set to .05 was used for between subject analyzes, and Wilcoxon Signed Ranks Test for between subjects analyzes. 
Table 1. Dependent measures and their definitions

\begin{tabular}{|c|c|c|}
\hline & Measure & Definition \\
\hline \multirow{6}{*}{  } & Mean speed & Vehicle's mean speed \\
\hline & Standard deviation of speed & How much the vehicle's speed deviates \\
\hline & Maximum speed & Maximum speed reached \\
\hline & Standard deviation from speed limit & How much the vehicle's speed deviates from the posted speed limit \\
\hline & Standard deviation of lane position & How much the driver's lateral control of the vehicle deviates \\
\hline & Number of lane exceedences & The number of times more than half of the vehicle is exceeds the lane \\
\hline \multirow{4}{*}{  } & & \\
\hline & Mean GSR & Difference in Galvanic Skin Resistance while relaxed and while driving \\
\hline & Mean temp & Difference in mean hand temperature while relaxed and while driving \\
\hline & Mean HR & Difference in drivers mean Heart Rate while relaxed and while driving \\
\hline \multirow{7}{*}{  } & Time to notice & Time from a warning appears until gaze is directed towards display \\
\hline & Glance frequency & Number of glances to the warning while it is displayed \\
\hline & Total glance duration & Total time the warning display is gazed upon \\
\hline & Mean glance duration & Mean time the warning display is gazed upon \\
\hline & Gaze duration off road scene ahead & Time the gaze is not directed towards the road scene ahead \\
\hline & HUD duration & Total time spent viewing the Head-up display \\
\hline & HDD duration & Total time spent viewing the Head-down display \\
\hline & Task completio & The time from a warning appears until it is reset \\
\hline
\end{tabular}

\section{RESULTS}

Differences between driving tasks for "Redundant HUD”. Adding a simple secondary visual detection task to the driving task significantly increased the mean speed, $(z=-2.701, p=0.007)$, while the standard deviation of speed was significantly lower during "driving with task", $(z=-$ $2.191, p=0.028)$. No significant differences were found regarding the physiological measures.

Differences between driving tasks for "Spread out". The "driving only" condition showed lower means for HDD duration, $(z=-2.31, p=0.021)$, compared to "driving with task". No significant differences were found regarding the driving performance or physiological measures.

Differences between display configurations for "driving only". When comparing "driving only" data for the two display configurations, the "Redundant HUD" showed a significantly lower mean for standard deviation of lane position, $(U=102.000, p=0.023)$. "Redundant HUD" also showed a lower mean for HDD duration, $(U=54.500, p=0.000)$, and a higher mean for HUD duration, $(U=94.000, p=0.012)$, caused by the HUD-speedometer. There were no significant differences between the display configurations and gaze duration off road scene ahead or any of the physiological measures.

Differences between display configurations for "driving and task". Data was analyzed for every separate warning occurrence from the time the warning appeared until $15 \mathrm{~s}$ after it was reset. This time interval was chosen because the driving performance and the physiological measures were hypothesized to also be affected a short while after the completion of the secondary task. When analyzing all warning occurrences together, the "Redundant HUD" showed significantly lower means for: standard deviation of lane position, $(U=17.000, p=0.022)$, and number of lane exceedences, $(U=11.000, p=0.005)$. In total 45 lane exceedences occurred for the "Redundant HUD" and 68 for the "Spread out". The glance measures showed lower means for "Redundant 
HUD" for: time to notice, $(U=21.000, p=0.050)$, glance frequency, $(U=7.500, p=0.002)$, total glance duration, $(U=19.000, p=0.034)$, Gaze duration off road scene ahead, $(U=11.000$, $p=0.006)$, and HDD duration, $(U=6.500, p=0.002)$. "Redundant HUD" showed a higher mean for HUD duration, $(U=14.500, p=0.013)$. The secondary task was performed faster for the "Redundant HUD" than for the "Spread out", $(U=16.000, p=0.018)$. Detection times were analysed for each separate warning in order to study if warnings are distinguished from normal in-vehicle information (Table 4). One warning occurrence did stand out for the "Spread out"; namely warning 10 which was displayed in the centerstack position during a high workload traffic environment (Table 4). Significant lower detection times was found for the "Redundant HUD” for warning 2, $(U=15.000, p=0.026)$, and warning $10,(U=12.000, p=0.007)$. For "Redundant HUD", the participants had the opportunity to choose which one of the HUD and HDD displays to consult to recognize a warning. Seven out of ten participants chose to exclusively consult the HUD, one checked the HUD for nine of the ten warning occurrences, and two preferred the HDD prior to the HUD for eight of the warnings.

Table 2. Mean detection times for each warning (in seconds)

\begin{tabular}{l|cccccccccc}
\hline Warning number & 1 & 2 & 3 & 4 & 5 & 6 & 7 & 8 & 9 & 10 \\
\hline Time to notice "Redundant HUD" & 1.17 & 2.24 & 2.22 & 1.00 & 1.32 & 3.04 & 1.56 & 4.89 & 5.43 & 0.95 \\
Time to notice "Spread out" & 4.28 & 3.68 & 1.98 & 0.90 & 4.20 & 3.13 & 2.54 & 3.27 & 3.34 & 18.16
\end{tabular}

\section{DISCUSSION}

The results in this study did show some differences between the two display configurations, which are in line with earlier research, namely that the display configuration with more centralized information resulted in better driving- and task performance than the spread out display configuration (Horrey, Wickens, \& Consalus, 2005; Wittman et al., 2006). According to Tsimhoni and Green (2001), the addition of a secondary task while driving significantly impairs driving performance, which could not be confirmed in this study. Probably due to the simplicity of the secondary task used which was not sufficient to cause extra workload to yield any differences in lane keeping or stress. Some differences were, however, found regarding speed maintenance in the "Redundant HUD", where the speed varied even less when the task was performed. This could be explained by the HUD-speedometer; at the same time the warning was gazed upon, the vehicle's speed was also monitored. More time was spent looking at the HDD when a task was added for "Spread out". However, this result needs further investigation to be explained. Both driving tasks rendered similar results in favour of the "Redundant HUD". Even though much time is spent looking at the HUD for "Redundant HUD", the driving performance is not negatively effected, but instead improved. This is probably an effect of the increased time the road is viewed compared to the "Spread out". Looking down at the HDD and the other display positions is more time consuming than looking at the HUD. The results also imply that despite the highly salient position of "Redundant HUD" close to the driver's field of view, this does not attract unnecessary attention as the glance duration towards the "Redundant HUD" was lower. Since it is desirable to keep the eyes on the road, the fewer glances towards the vehicle interior should be the better. "Spread out" had more glances away from the road, which causes the driver to get "out of the driving loop" more often (Kircher, 2007) and, thus, could have a negative effect on safety. The lesser lane exceedences for "Redundant HUD" than for "Spread 
out" during "driving and task" also imply that looking too much at in-vehicle displays has a detrimental effect on driving safety. There was no problem for the drivers to notice the warnings when they were displayed, except from for the CS position. Hence, this position should clearly be avoided when presenting emergency information to the driver, as can be seen in Table 4. For warning occurrences 2 and 10, where the warning was displayed in the CS position for "Spread out", the detection time was significantly higher. It is noticeable that the HUD was the preferred display to observe in "Redundant HUD". None of the participants had a HUD in their personal vehicle so the HUD was perhaps not naturally the primary choice in the search for information. Although, the highly salient position of the very novel HUD technology would ensure that presented information easily catch the driver's eye, but not so much that it would cause distraction.

The physiological measures did, however, not imply any differences between the two display configurations or between the driving tasks. The stress caused by the display configuration and/or the task was probably too small to be detected compared to the overall stress caused by driving the simulator or the physiological measures used might not be suitable for evaluating invehicle systems. Nevertheless, stress could be of interest to study if the stress level caused by the driving situation can be filtered out from the stress caused by in-vehicle systems. If the trials had lasted longer, the participants' stress and vigilance levels might have been lower, which could have made it easier to detect any differences. Although the ten warnings presented to the driver were generic, and the task was to off-set the warnings as soon as they were noticed, there was a possibility that the warnings were perceived as different from each other. However, this should not have any major effect on the results since the participants were told to take action as soon as a warning was presented. The participants seemed to remain highly vigilant during the driving blocks and in some cases repeatedly scanned the vehicle interior for new warnings, which, on the other hand, could have effected the time it took to notice a warning, but, if so, it should have affected both display configurations equally.

This study showed that even though the task of detecting and off-setting a warning is quite simple, it can still be used to reveal decreased driving- or task performance caused by in-vehicle technologies or vehicle interior design. This study is to be extended with a more demanding task in order to find out if the nature of the task causes different impact on driver performance and driving safety.

\section{ACKNOWLEDGMENTS}

This study was sponsored by the OPTIVe (OPTimized system integration for safe Interaction in Vehicles) project within the Swedish IVSS research foundation.

\section{REFERENCES}

Baber, C., \& Wankling, J. (1992). An experimental comparison of text and symbols for in-car reconfigurable displays. Applied Ergonomics, 23(4), 255-262.

Carsten, O.M.J., Merat, N., Janssen, W.H., Johansson, E., Fowkes, M., \& Brookhuis, K.A., (2005). HASTE Final Report. Institute for Transportation Studies, University of Leeds. 
Ellis, S.R. (2005). On redundancy in the design of spatial instruments In: Proceedings of the 49th Annual Meeting of the Human Factors and Ergonomics Society (pp.1561-1564). Santa Monica, CA: HFES.

Gish, K.W., \& Staplin, L., 1995. Human Factors Aspects of Using Head-Up Displays in automobiles: A Review of the Literature (Interim Rep. DOT HS 808 320). National Highway Traffic Safety Administration, Washington, DC.

Horberry, T., Anderson, J., Regan, M.A., Triggs, T.J., \& Brown, J. (2006). Driver distraction: The effects of concurrent in-vehicle tasks, road environment complexity and age on driving performance Accident Analysis and Prevention, 38(1), 185-191.

Horrey, W.J., Wickens, C.D., \& Consalus, K.P. (2005). The distracted driver: Modeling the impact of information bandwidth, in-vehicle task priority, and spatial-separation on driver performance and attention allocation (Tech. Rep. No. AHFD-05-11/GM-05-2). Savoy, IL: University of Illinois, Aviation Human Factors Division.

Horrey, W.J., \& Wickens, C.D. (2007). In-Vehicle Glance Duration. Distributions, Tails, and Model of Crash Risk. Transportation Research Record: Journal of the Transportation Research Board, 2018, 22-28.

Johansson, E., Engström, J., Cherri, C., Nodari, E., Toffetti, A., Schindhelm, R. et al. (2004) Deliverable 2.2.1 - Review of existing techniques and metrics for IVIS and ADAS assessment. AIDE Project IST-1-507674-IP.

Liu, Y.-C., \& Wen, M.-H. (2004). Comparison of head-up display (HUD) vs. head-down display (HDD): driving performance of commercial vehicle operators in Taiwan. International Journal of Human-Computer Studies, 61, 679-697.

Luoma, J., \& Rämä, P. (2002). Acceptance of traffic sign information provided by an in-vehicle terminal. In: Proceedings of the Ninth World Congress on Intelligent Transportation Systems. Washington, DC: ITS America.

Kircher, K. (2007). Driver distraction: A review of the literature (VTI rapport R594A) Linköping: VTI.

Noy, Y. I. (1997). Human factors in modern traffic systems. Ergonomics, 40 (10), 1016-1024.

Seagull, F.J., Wickens, C.D., \& Loeb., R.G. (2001). When less is more? Attention and workload in auditory, visual, and redundant patient monitoring conditions. In: Proceedings of the 45th Annual Meeting of the Human Factors and Ergonomics Society (pp. 1395-1399). Santa Monica, CA: HFES.

Tsimhoni, O., \& Green, P. (2001). Visual demand of driving and time execution of displayintensive in-vehicle tasks. In: Proceedings of the Human Factors and Ergonomics Society 45th Annual Meeting (pp. 1586-1590) Santa Monica, CA: HFES.

Wittman, M., Kiss, M., Gugg, P., Steffen, A., Fink., Pöppel, E. et al. (2006). Effects of display position of a visual in-vehicle task on simulated driving. Applied Ergonomics, 37(2006), 187199. 